\title{
TREINAMENTO FUNCIONAL: CONCEITOS E BENEFÍCIOS
}

\section{ARTIGO DE REVISÃO}

ANTUNES, Bianca Siqueira ${ }^{1}$

BIANCO, Roberto ${ }^{2}$

LIMA, Wilson Pereira ${ }^{3}$

ANTUNES, Bianca Siqueira. BIANCO, Roberto. LIMA, Wilson Pereira. Treinamento funcional: Conceitos e benefícios. Revista Científica Multidisciplinar Núcleo do Conhecimento. Ano 05, Ed. 06, Vol. 08, pp. 69-80. Junho de 2020. ISSN: 2448-0959, Link de acesso: https://www.nucleodoconhecimento.com.br/educacaofisica/treinamento-funcional

\section{RESUMO}

Este estudo discute as características e os benefícios que podem ser proporcionados pela prática do treinamento funcional. A ideia central abordada tem como base estudos de diferentes autores acerca do assunto, ocasionando diversas visões a respeito dos conceitos existentes de treinamento funcional. $O$ treinamento funcional foi o tipo de treino escolhido para esta investigação, tendo em vista seu desempenho crescente ao longo dos anos. Para esta análise foi realizada uma revisão bibliográfica.

${ }^{1}$ Pós Graduada em Cinesiologia, Biomecânica e Treinamento Físico - Universidade Estácio de Sá. Pós Graduada em Educação Física Escolar - Universidade Estácio de Sá. Graduada em Bacharelado em Educação Física - Universidade Estácio de Sá. Graduada em Licenciatura em Educação Física - Universidade Federal do Rio de Janeiro.

${ }^{2}$ Doutorado em Ciência. Mestrado em Educação Física. Graduação em Bacharelado em Educação Física.

${ }^{3}$ Mestrado em andamento em Educação Física. Especialização em Biomecânica da Atividade Física e Saúde. Graduação em Educação Física. 
As informações encontradas permitem concluir que a maioria dos autores tem ideias convergentes e que se complementam, acerca do conceito e das características do treinamento funcional. Sobre os benefícios, todos apontam para melhoras em nível de bem-estar, saúde, performance e eficiência nas atividades diárias.

Palavras-chave: Treinamento funcional, características, benefícios.

\section{INTRODUÇÃO}

De acordo com Boyle (2015) o treinamento funcional pode ser descrito como um treinamento com um propósito, já que função é essencialmente o propósito. Teixeira e Guedes Júnior (2010) afirmam que o treinamento funcional se baseia no conceito da especificidade, pois é caracterizado pela semelhança do exercício com situações do dia a dia.

É explícito o grande avanço desse estilo de treino nos últimos anos, que o fez alcançar notoriedade em nível mundial e vem conquistando muito espaço na rotina de indivíduos de diferentes faixas etárias e classes sociais. Porém, grande parte das pessoas possui uma visão distorcida do que realmente se trata o treinamento funcional, associando-o simplesmente a um treino com materiais diversos como bolas, rolos, elásticos, etc.

Com isso, o treinamento funcional fica descaracterizado, sem sentido algum, ficando difícil compreender para o que serve esse tipo de treino, e o que ele traria de benefícios para seus praticantes. Logo, percebe-se a importância de estudar a temática acerca dos efeitos e possibilidades desse treinamento, levando em consideração os conceitos apresentados inicialmente nesse estudo.

Diante do exposto o presente artigo tem como objetivo identificar os benefícios do treinamento funcional, bem como suas possibilidades práticas.

Dados os pressupostos iniciais, será utilizada neste estudo uma revisão de literatura em artigos e livros nacionais e internacionais, com o intuito de reunir os principais 
efeitos relativos à prática desse treinamento, além de subsidiar teoricamente profissionais da educação física neste contexto.

\section{REVISÃO DE LITERATURA}

\subsection{CONCEITOS E CARACTERÍSTICAS DO TREINAMENTO FUNCIONAL}

Como toda novidade, o treinamento funcional despertou o interesse de profissionais da área e passou a ser incluído nas academias de ginástica. Assim como, ganhou vários conceitos diferentes sobre o que de fato é, conceitos esses que às vezes são criticados por alguns autores e reformulados por outros.

O dicionário de língua portuguesa mostra que treinamento é definido como a ação de treinar, e funcional refere-se às funções vitais. Então, ao fazer a associação de palavras, treinamento funcional é a ação de treinar para aprimorar as funções vitais. (TEIXEIRA e EVANGELISTA, 2014).

De acordo com Teixeira e Guedes Júnior (2010) o termo "funcional" só era utilizado antigamente para fins de reabilitação, mas com o tempo passou a ser incluído nas práticas de exercícios de muitos alunos. Como todo treinamento, o funcional vem para somar às demais estratégias de treinamento e possibilitar um desenvolvimento corporal mais homogêneo.

Segundo Novaes, Gil e Rodrigues (2014) o termo treinamento funcional originou-se com profissionais da fisioterapia e reabilitação que trabalhavam com a finalidade de devolver a funcionalidade de suas atividades habituais a seus pacientes lesionados. Nos últimos anos os profissionais da área de educação física têm inserido esse tipo de treino para agregar benefícios ao praticante, e o definem como um treino cuja finalidade é refinar a capacidade funcional do corpo, sendo para isso necessário o treinamento da região central do corpo, definida como "core". 
Staley (2005) define o treinamento funcional como exercícios realizados em vários aparatos elaborados para criar um ambiente desafiador para envolver o sistema neuromuscular. Ainda diz que aqueles que propõem esse tipo de treino insistem em afirmar que esse tipo de exercício trabalha mais os músculos estabilizadores menores e mais profundamente localizados, argumento que ele não concorda.

Boyle (2015) afirma que treinamento funcional e treinamento em superfície instável não são sinônimos. Para o autor, função significa propósito, logo, pode ser descrito como um treinamento com um propósito. O que de fato o treinamento funcional faz é desviar o foco do exercício para incorporar os músculos estabilizadores, pois neles encontram-se a fonte das lesões, segundo os fisioterapeutas.

Liebenson (2017) considera que o treinamento funcional se torna mais funcional na medida em que o foco passa a ser desenvolver padrões de movimentos estereotípicos que um atleta usa em todos os esportes, em vez de movimentos isolados. Então, se o objetivo for melhorar a qualidade para tarefas funcionais, o movimento integrado deve ser o foco.

Bossi (2013) diz que o treinamento funcional deve ser parte de um treinamento envolvendo trabalho de força, hipertrofia e resistência muscular localizada, como preparação para o treino funcional. Considera como um problema atual a busca pela funcionalidade e equilíbrio que ocasiona uma redução expressiva da carga envolvida no treino, a fim de trabalhar a musculatura estabilizadora, e, por fim, causando um destreinamento na musculatura agonista.

Segundo Silva-Grigoletto et al. (2014) para um treinamento ser considerado funcional deve contemplar exercícios selecionados tendo como critério a sua funcionalidade e para isto deve-se atender às cinco variáveis distintas da funcionalidade, que são: frequência dos estímulos, volume de cada sessão, intensidade adequada, densidade e organização metodológica das tarefas.

Resende-Neto et al. (2016) afirmam que atualmente o conceito de treinamento funcional é definido pela estruturação de programas de treinamento para o sistema 
neuromuscular com o uso de exercícios e movimentos considerados funcionais para as necessidades específicas da vida diária.

Clark (2001 apud MONTEIRO e EVANGELISTA, 2015) aponta que movimentos funcionais são movimentos integrados, multiplanares, que envolvem redução, estabilização e produção de força, buscando a melhora dos componentes necessários que permitem ao praticante adquirir um nível ótimo de função. Além disso, flexível e ilimitado, já que é possível realizar movimentos em amplitudes diversas, com infinitas variações

D'Elia (2017) considera o conceito de treinamento funcional como formar uma base de exercícios mais amplos, com o intuito de gerar especificidades para demandas neurais, mecânicas e metabólicas em conjunto, para a melhora da performance.

De acordo com Teixeira et al. (2016) a principal característica do treinamento funcional é o objetivo, pois enquanto que no treino tradicional há uma tendência de se objetivar a estética, no funcional objetiva-se a função, tendo como norteador o princípio da especificidade, assumindo as características das atividades cotidianas, sendo integrado, assimétrico, acíclico e multiplanar. Outra característica é a estimulação da adaptação do sistema de controle e coordenação do movimento humano.

Cress et al. (1996) são bem objetivos ao falar sobre a importância da especificidade do treinamento citando exemplos como: nadadores devem treinar nadando, levantadores de peso devem levantar pesos, jogadores de futebol americano devem correr e arremessar. Questionam que apesar dessa estratégia funcionar para atletas, não é usada nos treinamentos diários de um adulto, em que a intenção seria justamente desenvolver as funções utilizadas nas atividades diárias. Para isso, é necessário um treino que promova a integração de múltiplas articulações e vários grupos musculares, já que nos nossos movimentos do dia a dia realizamos sempre ações coordenadas.

Liu et al. (2014) também citam que a base do treinamento funcional é a especificidade do treinamento, o que significa que treinar especificamente para aquilo que se quer 
melhorar é a melhor maneira de consegui-lo. Sobre as características do treino funcional, destacam a necessidade de trabalhar a musculatura de forma integrada, em movimentos multiplanares e dinâmicos.

O treinamento funcional vem aparecendo como um método inovador, que prioriza a capacidade funcional do indivíduo, com uma abordagem dinâmica, motivante, desafiadora e complexa. $\mathrm{O}$ treino tem como base movimentos do cotidiano, com o intuito de treinar o indivíduo para desempenhar, com eficiência, suas tarefas diárias. É caracterizado como um exercício contínuo que envolve equilíbrio e propriocepção através da estabilização do core. (ANDRADES e SALDANHA, 2012)

Moreira et al. (2011) acreditam que um aspecto de vital importância nesse tipo de treino é a utilização de exercícios que estimulem a propriocepção, em materiais que oferecem superfícies instáveis com movimentos realizados em vários planos e eixos. Através de exercícios que desafiam os diversos componentes do sistema nervoso, o treinamento funcional visa a melhoria dos aspectos neurológicos que afetam a funcionalidade do corpo humano.

D’Elia e D’Elia (2005) consideram que o treinamento funcional representa uma nova forma de condicionamento, e afirmam que o aparecimento do treinamento funcional se deu em função de três pontos fundamentais, sendo eles o maior volume de informação que o praticante de atividade física recebe hoje em dia; a mudança do padrão estético vigente, aliando agora boa forma física e performance; a estagnação do modelo de atividade física que academias, clubes e escolas apresentam.

O treinamento funcional tem como objetivo melhorar a capacidade funcional, através de exercícios que estimulam os receptores proprioceptivos. Com isso é proporcionado o desenvolvimento da consciência sinestésica e do controle corporal, do equilíbrio muscular estático e dinâmico, além de diminuir a incidência de lesão e aumentar a eficiência dos movimentos. (LEAL et al., 2009)

Com o avanço dessa investigação acerca dos conceitos e características do treinamento funcional, é possível perceber que a maioria dos autores possuem ideias 
semelhantes e que muitas vezes se complementam para a definição de treinamento funcional. E já que se tornou uma modalidade bastante praticada, tem-se a necessidade de analisar a seguir, os benefícios que podem ser adquiridos com sua prática.

\subsection{BENEFÍCIOS}

Um dos pontos mais relevantes a respeito do treinamento funcional trata-se dos benefícios por ele oferecidos. Para começar a trabalhar e praticar exercícios funcionais é necessário que o indivíduo deve possuir músculos e articulações preparados para suportar ações musculares e equilíbrio exigidos. (BOSSI, 2013)

Com base nos resultados da pesquisa de Resende-Neto et al. (2016) o treinamento funcional é uma alternativa segura que traz impactos positivos sobre a massa muscular, força e potência muscular, resistência cardiorrespiratória, flexibilidade, equilíbrio e cognição. Apesar disso, ainda há carência de um modelo sistematizado para uma melhor comparação entre protocolos utilizados.

O estudo de Leal et al. (2009) atingiu o objetivo inicial, que era melhorar a autonomia funcional, equilíbrio e qualidade de vida, sugerindo melhora no desempenho das atividades da vida diária.

Segundo Silva-Grigoleto et al. (2014), se a prescrição do treinamento seguir uma fundamentação, e as variáveis desse treino foram controladas e manipuladas de forma com que se concretize uma dose adequada de exercício que o indivíduo deve realizar, o estado psicobiológico será adequadamente estimulado, gerando respostas e adaptações positivas.

Tendo em vista o exposto por Novaes, Gil e Rodrigues (2014) o treinamento funcional aperfeiçoa todas as aptidões do sistema musculoesquelético refletindo melhoria nas atividades cotidianas e nos gestos esportivos específicos. Promove também benefícios ao bem-estar, saúde, estética e desempenho, propiciando o equilíbrio entre a estabilidade central do corpo (core), o controle neuromuscular e neuromotor. 
Conforme Andrades e Saldanha (2012), que pesquisaram a respeito dos benefícios para o equilíbrio com o treinamento funcional, pode-se observar que como o treino funcional trabalha outras capacidades além da força, é notória a evolução em termos de melhora do equilíbrio no grupo analisado. Além disso, força, velocidade, coordenação, flexibilidade e resistência também apresentam desenvolvimento e proporcionam ganhos significativos de performance para o indivíduo.

Segundo Boyle (2015), o treinamento funcional desvia o foco dos exercícios para incorporar os músculos estabilizadores, o que beneficiaria na diminuição de incidência de lesões. A técnica e a qualidade são de extrema importância para o recrutamento da musculatura estabilizadora, e para permitir os ganhos que o treino funcional proporciona.

Cress et al. (1996) relataram que o propósito da pesquisa era avaliar os benefícios do treinamento funcional em mulheres mais velhas, com um exercício de subida de escada, com carga extra, além de um trabalho aeróbio, já que são atividades comuns nas rotinas das pessoas. Foi observado que ocorreram adaptações nas fibras musculares, com crescimento da força, que foi considerado como um desenvolvimento total da performance do corpo, o que é bom, já que se trata de uma tarefa importante para uma vida independente.

Os estudos de Liu et al. (2014) demonstrou resultados benéficos na força muscular, equilíbrio, mobilidade e nas atividades da vida diária, principalmente quando o treino era especificamente voltado para isso. Concluiu-se que o treinamento funcional pode ser utilizado para melhorar a performance de idosos, e pode ser uma opção melhor do que o treinamento de força por si só, se o objetivo for diminuir a incapacidade do idoso de realizar alguma tarefa de sua rotina.

Atualmente, os adultos passam a maior parte do tempo realizando tarefas referentes ao trabalho, tarefas essas consideradas laborais, envolvidas com automação. Além disso, é observado que há diminuição da prática de atividades físicas na infância e na adolescência, justamente a fase que se desenvolvem habilidades motoras, devido ao 
avanço da tecnologia e ao aumento da violência. (MONTEIRO E EVANGELISTA, 2015)

Com base nesses aspectos, pode ocorrer o desenvolvimento de desequilíbrios musculares e diminuição da capacidade total de trabalho, já que nem os adultos nem as crianças e adolescentes estão estabelecendo boa eficiência neuromuscular, flexibilidade dinâmica e força funcional, além de o número de disfunções e lesões estarem aumentando. Então, acredita-se que com a inserção de programas funcionais na vida dessas pessoas, haveria benefícios relativos a essas capacidades, que proporcionariam uma melhora no cotidiano. (MONTEIRO E EVANGELISTA, 2015)

$\mathrm{Na}$ pesquisa de Moreira et al. (2011) foi feita uma comparação entre os benefícios obtidos pelos idosos através do treinamento funcional resistido e do treinamento de força. Foram observadas melhoras na condição física geral do idoso devido à reabilitação da saúde, além da prevenção de determinadas patologias. Isso facilita a manutenção de bons níveis de independência e autonomia para as atividades da vida diária.

Segundo Teixeira e Evangelista (2014), como o treinamento funcional contempla capacidades físicas distintas, já que envolvem combinações de exercícios neuromotores, de resistência e de flexibilidade, os benefícios surgem em nível de melhora da habilidade e capacidade funcional para a realização de funções cotidianas e/ou esportivas com autonomia e segurança.

\section{DISCUSSÃO}

Objetivando identificar os benefícios do treinamento funcional, bem como suas possibilidades práticas, foi feita, neste artigo, uma revisão de literatura com o intuito de reunir as principais características e os benefícios relativos à prática desse treinamento. Foi possível perceber muitas ideias semelhantes entre os pesquisadores da área, com uma ou outra divergência a respeito de determinado aspecto. 
Tanto Teixeira e Guedes Júnior (2010) como Novaes, Gil e Rodrigues (2014) abordam o conceito de treinamento funcional com base no fato dele ter-se originado para fins de reabilitação na fisioterapia, e com o tempo, ter passado a ser incluído nas práticas de exercícios de muitos alunos para possibilitar um desenvolvimento corporal mais homogêneo, refinando a capacidade funcional do corpo.

Convergem nas ideias acerca do assunto Liebenson (2017) e Monteiro e Evangelista (2015), que apontam movimentos funcionais como movimentos integrados em vez de isolados, que permitem ao praticante adquirir melhor qualidade em nível de função. Assim como Teixeira e Evangelista (2004) concordam que funcional é a ação de treinar para aprimorar as funções vitais.

Teixeira et al. (2016) e Liu et al. (2014) apontam que a principal característica do treino funcional é o objetivo, sendo baseado na especificidade, isto é, trabalhar especificamente aquilo que se quer melhorar nas atividades cotidianas.

Andrades e Saldanha (2012), Moreira et al. (2011) e Leal (2009), na hora de caracterizar o treinamento funcional, atentam para a utilização da propriocepção e do equilíbrio visando melhorar aspectos neurológicos e aumentar a eficiência de movimentos. Em contra partida, Bossi (2013) considera um problema essa busca pela funcionalidade e equilíbrio, pois ocasiona a redução expressiva da carga envolvida no treino, podendo causar um destreinamento na musculatura agonista.

Silva-Grigoleto et al. (2014), Resende-Neto et al. (2016), D'Elia (2017) e Cress et al. (1996) consideram que o treino funcional deve atender à variáveis distintas, definidas pela estruturação do treinamento, que contemplem demandas neurais, metabólicas e mecânicas em conjunto, coordenadas e integradas.

Visões divergentes são apresentadas nos trabalhos de Boyle (2015) e Staley (2005). Esse relaciona o treinamento funcional com exercícios realizados em vários aparatos elaborados para criar um ambiente desafiador. Aquele diz que treinamento funcional, e treinamento em superfícies instáveis não são sinônimos, indo de encontro às ideias de Staley. 
Em termos de benefícios proporcionados pela prática dessa atividade, entende-se que todos os autores apontam benefícios em nível de bem-estar, saúde e performance, principalmente aquela relacionada às atividades do dia a dia.

\section{CONSIDERAÇÕES FINAIS}

O presente estudo identificou conceitos e características do treinamento funcional, definidos por diversos autores nos últimos anos, bem como os benefícios adquiridos pela prática desse, tanto por jovens, como adultos e idosos.

Tendo em vista o exposto acima, é possível perceber que a maioria das ideias de cada autor se complementam para formar uma visão mais ampla acerca das possibilidades do treinamento funcional.

Como resultado de análise de diferentes autores, pode-se concluir que o treinamento funcional é um método eficiente que contribui para a evolução de habilidades utilizadas no cotidiano ou para determinada atividade específica, além de ajudar no desenvolvimento de outras capacidades físicas, como força, flexibilidade, equilíbrio, resistência muscular, entre outras. Uma atividade que deve envolver o trabalho do corpo em sua totalidade, respeitando os limites de cada um, baseando-se na funcionalidade e especificidade, para alcançar resultados positivos.

\section{REFERÊNCIAS}

ANDRADES, Michele Taís de; SALDANHA, Ricardo Pedrozo. Treinamento funcional: o efeito da estabilização do core sobre o equilíbrio e propriocepção de mulheres adultas saudáveis e fisicamente ativas. Revista Vento e Movimento, v. 1, n. 1, p. 114-133, 2012.

Disponível em: http://facos.edu.br/publicacoes/revistas/vento_e_movimento/abril_2012/\#/page/1

BOSSI, Luis Cláudio. Treinamento funcional da musculação. São Paulo: Phorte, 2013.

BOYLE, Michael. Avanços no treinamento funcional. Porto Alegre: Artmed, 2015. 
CRESS, M. Elaine; CONLEY, Kevin E.; BALDING, Sheryal L.; HANSEN-SMITH, Fay; KONCZAK, Jürgen. Functional training: muscle structure, function, and performance in older women. J. Orthop. Sports Physical Ther., v. 24, n. 1, p. 4-10, 1996. Disponível em: https://www.jospt.org/doi/abs/10.2519/jospt.1996.24.1.4

D’ELIA, Luciano. Guia completo de treinamento funcional. São Paulo: Phorte, 2017.

D'ELIA, R.; D'ELIA, L. Treinamento funcional: 6o treinamento de professores e instrutores. São Paulo: SESC - Serviço Social do Comércio, 2005. Apostila.

LEAL, Silvânia Matheus de Oliveira; BORGES, Eliane Gomes da Silva; FONSECA, Marília Andrade; ALVES JUNIOR, Edmundo Drumond; CADER, Samária; DANTAS, Estélio Henrique Martins. Efeitos do treinamento funcional na autonomia funcional, equilíbrio e qualidade de vida de idosas. R. bras. Ci. e Mov., v. 17, n. 3, p. 61-69, 2009. Disponível em: https://portalrevistas.ucb.br/index.php/RBCM/article/view/1045

LIEBENSON, Craig. Treinamento funcional na prática desportiva e reabilitação neuromuscular. Porto Alegre: Artmed, 2017.

LIU, Chiung-ju; SHIROY, Deepika M.; JONES, Leah Y.; CLARK, Daniel O. Systematic review of functional training on muscle strength, physical functioning, and activities of daily living in older adults. Eur Rev Aging Phys Act., v. 11, p. 95-106, 2014. Disponível em: https://link.springer.com/article/10.1007/s11556-014-0144-1

MONTEIRO, Artur Guerrini, EVANGELISTA, Alexandre Lopes. Treinamento funcional: uma abordagem prática. 3. ed. São Paulo: Phorte, 2015.

MOREIRA, Josiana Kely Rodrigues; CRUZ, Raiza da Costa Santos; NAVES, Samira Cristiane Teixeira; NASCIMENTO, Vanderson Cunha do; SOUSA, Evitom Corrêa de. Treinamento de força e treinamento funcional resistido sobre a capacidade funcional e qualidade de vida de idosos fisicamente independentes. Fiep Bulletin, v. 81, special edition, 2011.

Disponível em:

http://www.fiepbulletin.net/index.php/fiepbulletin/article/download/352/651 
NOVAES, Jefferson; Gil, Ana; RODRIGUES, Gabriel. Condicionamento físico e treino funcional: revisando alguns conceitos e posicionamentos. Revista Uniandrade, v. 15, n. $2, \quad$ p. $87-93,2014 . \quad$ Disponível em: https://www.uniandrade.br/revistauniandrade/index.php/revistauniandrade/article/vie w/136

RESENDE-NETO, Antônio Gomes; SILVA-GRIGOLETTO, Marzo Edir da; SANTOS, Marta Silva; CYRINO, Edilson Serpeloni. Treinamento funcional para idosos: uma breve revisão. R. bras. Ci. e Mov., v. 24, n. 3, p. 167-177, 2016. Disponível em: https://portalrevistas.ucb.br/index.php/RBCM/article/viewFile/6564/pdf

SILVA-GRIGOLETTO, Marzo Edir da; BRITO, Ciro Jose; HEREDIA, Juan Ramon. Treinamento funcional: funcional para que e para quem? Rev Bras Cineantropom Desempenho Hum, v. 16, n. 6, p. 714-719, 2014. Disponível em: https://www.scielo.br/pdf/rbcdh/v16n6/pt_1980-0037-rbcdh-16-06-00714.pdf

STALEY, Charles. Muscle logic: escalating density training. Emmaus, Pa.: Rodale Books, 2005.

TEIXEIRA, Cauê La Scala; EVANGELISTA, Alexandre Lopes. Treinamento funcional e core training: definição de conceitos com base em revisão de literatura. EFDeportes.com, Revista Digital, Buenos Aires, ano 18, n. 188, jan. 2014. Disponível em: http://www.efdeportes.com/efd188/treinamento-funcional-e-coretraining.htm

TEIXEIRA, Cauê Vazquez La Scala; EVANGELISTA, Alexandre Lopes; PEREIRA, Cássio Adriano; SILVA-GRIGOLETTO, Marzo Edir da. Short roundtable RBCM: treinamento funcional. R. bras. Ci. e Mov., v. 24, n. 1, p. 200-206, 2016. Disponível em: https://portalrevistas.ucb.br/index.php/RBCM/article/view/6180/4275

TEIXEIRA, Cauê Vazquez La Scala.; GUEDES JUNIOR, Dilmar Pinto. Musculação perguntas e respostas: as 50 dúvidas mais frequentes nas academias. São Paulo: Phorte, 2010. 
Enviado: Maio, 2020.

Aprovado: Junho, 2020. 